\title{
The use of fluorine-18 fluorodeoxyglucose positron emission tomography for imaging human motor neuronal activation in the brain
}

\author{
KISOO PAHK ${ }^{1}$, KUN-WOO PARK ${ }^{2}$, SUNG BOM PYUN ${ }^{3}$, JAE SUNG LEE ${ }^{4}$, SUNGEUN KIM ${ }^{1}$ and JAE GOL CHOE ${ }^{1}$ \\ Departments of ${ }^{1}$ Nuclear Medicine, ${ }^{2}$ Neurology and ${ }^{3}$ Physical Medicine and Rehabilitation, \\ Korea University Anam Hospital, Seoul 136-705; ${ }^{4}$ Department of Nuclear Medicine, Seoul \\ National University College of Medicine, Seoul 110-799, Republic of Korea
}

Received February 3, 2015; Accepted September 1, 2015

DOI: $10.3892 /$ etm.2015.2788

\begin{abstract}
The present study aimed to visualize human motor neuronal activation in the brain using fluorine-18 fluorodeoxyglucose positron emission tomography (FDG-PET), and to develop an FDG-PET procedure for imaging neuronal activation. A male volunteer underwent 20 min periods of rest and motor activation, whilst being assessed using FDG-PET on two consecutive days. The motor task, which involved repetitively grasping and releasing the right hand, was performed during the initial $5 \mathrm{~min}$ of the activation period. Subtraction of the rest period signal from the activation PET images was performed using the subtraction ictal single-photon emission computed tomography co-registered to magnetic resonance imaging method. The subtracted image detected activation of the contralateral (left) primary motor cortex, supplementary motor area, and ipsilateral (right) cerebellum. In the present study, FDG-PET detected significantly increased motor-associated activation of the brain in a subject performing a motor task.
\end{abstract}

\section{Introduction}

The performance of voluntary motor tasks is associated with the primary motor cortex of the brain (1). Furthermore, cerebral blood flow and glucose metabolism have been demonstrated to positively correlate with neuronal processes during neuronal transmission in a healthy brain (2).

Numerous methods, including computed tomography (CT), magnetic resonance imaging (MRI), and functional MRI have previously been used to image the motor cortex; however, limi-

Correspondence to: Professor Sungeun Kim, Department of Nuclear Medicine, Korea University Anam Hospital, 5-Ga Anam-Dong, Seoul 136-705, Republic of Korea

E-mail: seiong@korea.ac.kr

Key words: motor cortex, motor cortex activation, motor image, neuroimaging, fluorodeoxyglucose positron emission tomography tations of these approaches are associated with their inability to fully capture a functional view of the motor cortex, instead only capturing the anatomical structure or blood flow $(3,4)$.

Fluorine-18 fluorodeoxyglucose positron emission tomography (FDG PET) detects FDG accumulation in tissue that is proportional to the amount of glucose metabolism, and has been in use for $>35$ years (5) in the study of neurology, oncology and cardiology (6). FDG-PET is regarded as an effective imaging method for visualizing glucose metabolism during cerebral blood flow (7) and is currently the most accurate method for the evaluation of human brain metabolism (8); however, studies that have investigated the use of FDG-PET for imaging motor cortex activation are limited.

The present study aimed to demonstrate human motor-associated activation of the brain using FDG-PET, and to develop an FDG-PET protocol for imaging neuronal activation.

\section{Materials and methods}

Patient. A healthy 30-year-old right-handed male volunteered to participate in the present study at Korea University Anam Hospital (Seoul, Republic of Korea). Written informed consent was obtained from the patient. This study was approval by the ethics committee of the Korea University Anam Hospital.

Imaging time and motor task. On day 1, following a 20-min preparatory rest period, the patient underwent FDG-PET analysis. On day 2, after performing $20 \mathrm{~min}$ of motor activation, a second session of FDG-PET was conducted (Fig. 1). The motor task, which involved repetitively grasping and releasing the right hand, was performed during the initial $5 \mathrm{~min}$ of the motor activation period.

Image acquisition. Images were obtained using a PET-CT scanner (Gemini TF; Philips Healthcare, Cleveland, OH, USA). The volunteer fasted for $\geq 6 \mathrm{~h}$, and serum glucose levels were $<180 \mathrm{mg} / \mathrm{dl}$, prior to scanning. Image acquisition (20 min) was initiated $20 \mathrm{~min}$ following intravenous injection of $341 \mathrm{MBq}$ fluorine-18 FDG. CT scans were obtained followed by PET emission scans for $1 \mathrm{~min}$ per scan. The PET unit had an axial field of view of $18 \mathrm{~cm}$ and a spatial resolution of $4.4 \mathrm{~mm}$. A 


\author{
Rest ( $1^{\text {st }}$ day) \\ F-18 FDG Injection (341MBq)
}

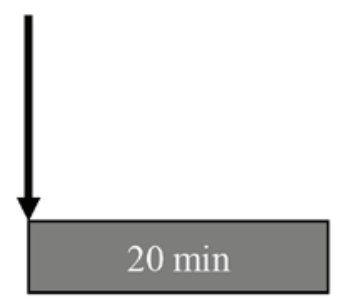

Scan Start

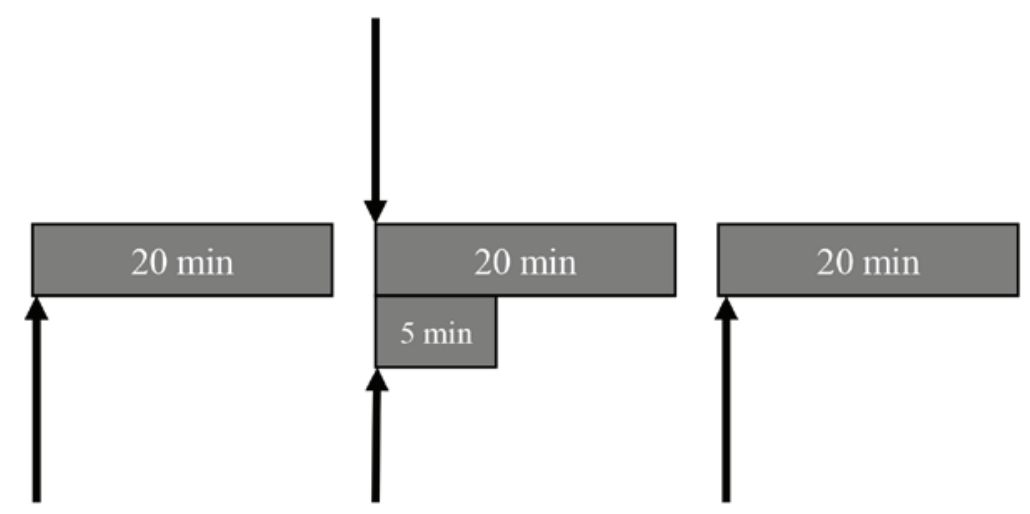

Activation (2nd $d a y)$

F-18 FDG Injection (341MBq)

Motor task during $5 \mathrm{~min}$

Scan Start

Figure 1. Schematic description of the 2-day F-18 FDG-positron emission tomography procedure. F-18 FDG, fluorine-18 fluorodeoxyglucose.

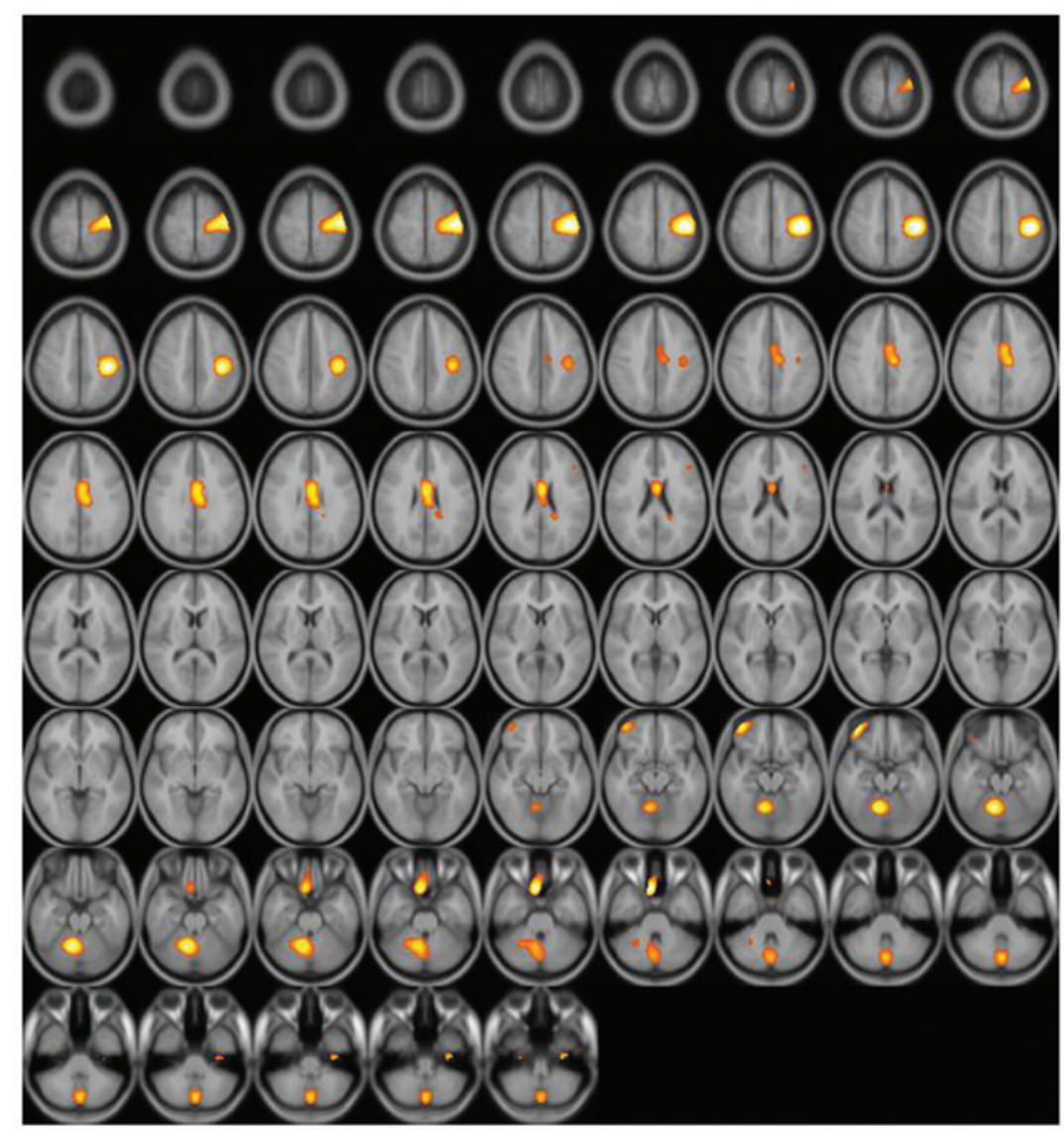

Figure 2. Subtraction positron emission tomography image: Axial view. Enhanced uptake of fluorine-18 fluorodeoxyglucose was observed in the supplementary motor area and ipsilateral cerebellum.

low-dose CT scan was obtained for attenuation correction and localization, with a 16-slice multidetector helical CT unit, using the following parameters: $120 \mathrm{kVp} ; 50 \mathrm{~mA} ; 0.75 \mathrm{sec}$ rotation time; $0.75 \mathrm{~mm}$ slice collimation; $2 \mathrm{~mm}$ scan reconstruction with a reconstruction index of $2 \mathrm{~mm} ; 60 \mathrm{~cm}$ field of view; and a $512 \times 512$ matrix. PET data were reconstructed iteratively using the 3 dimensional Row Action Maximum Likelihood Algorithm (9), with low-dose CT data-sets for attenuation correction. Maximum intensity projection, cross sectional views, and fusion images were generated and reviewed.

Image processing. Images were transformed and quantified using statistical probabilistic anatomical mapping, which is a well-established method for displaying functional brain images (10-12). Images were spatially normalized using the Statistical Parametric Mapping (SPM) 2 software (http:// 


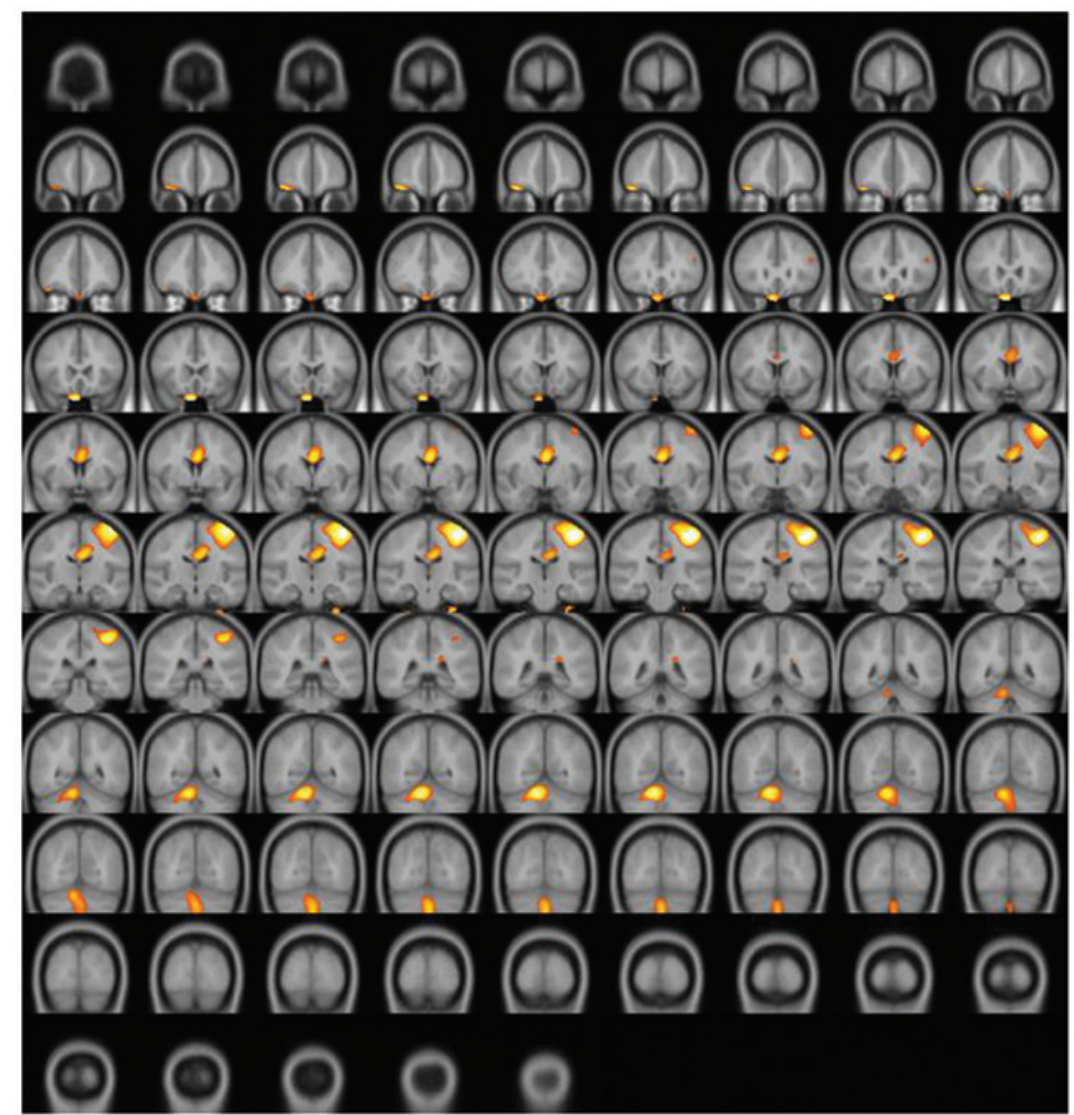

Figure 3. Subtraction positron emission tomography image: Coronal view. Enhanced uptake of fluorine-18 fluorodeoxyglucose was observed in the supplementary motor area and ipsilateral cerebellum.

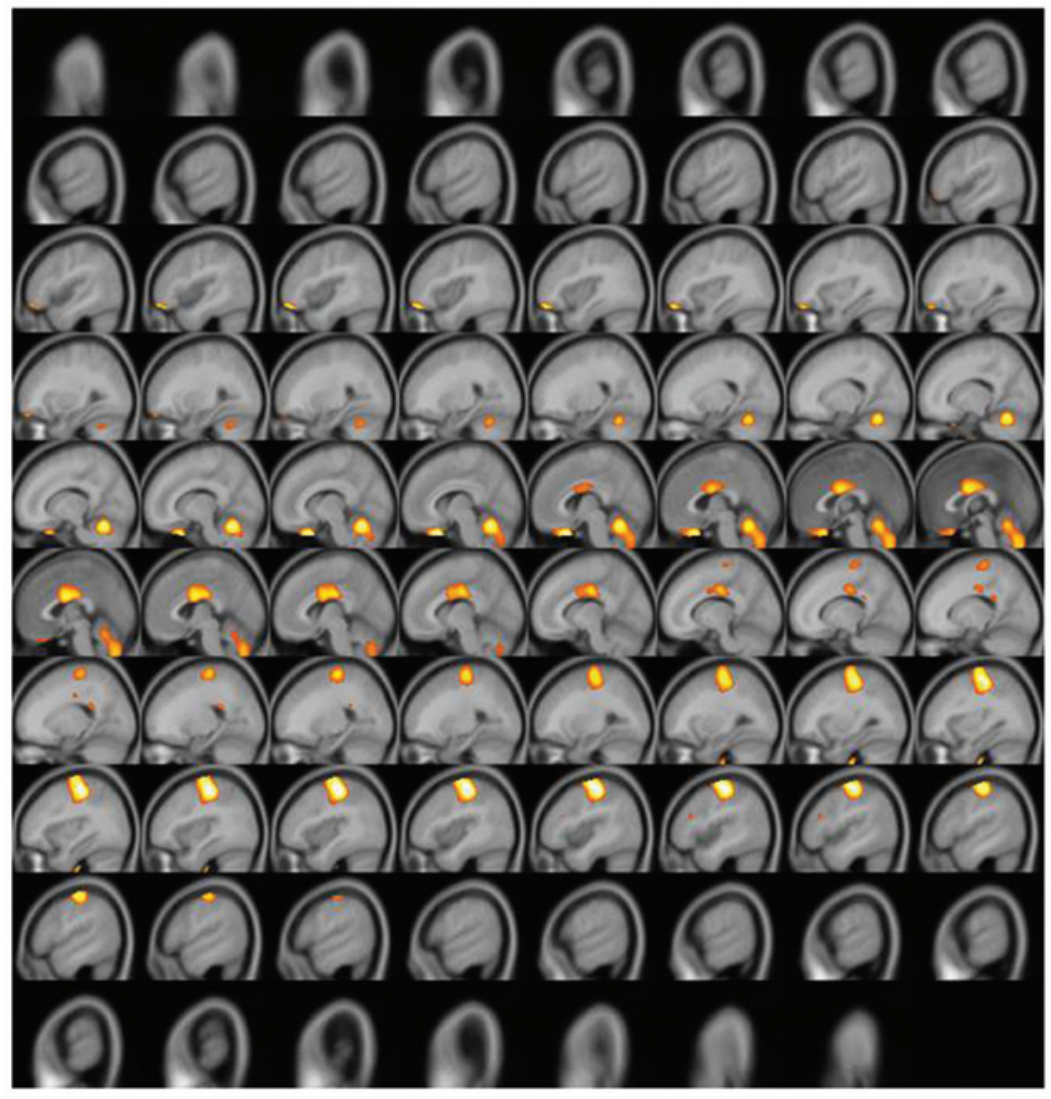

Figure 4. Subtraction positron emission tomography image: Sagittal view. Enhanced uptake of fluorine-18 fluorodeoxyglucose was observed in the supplementary motor area and ipsilateral cerebellum. 
www.fil.ion.ucl.ac.uk/spm/), according to the Korean standard templates provided by the Korean Structural Statistical Probabilistic Anatomical Map, implemented in Matlab 6.5 (13), which is based on the Korean standard brain atlas (13). Normalized images were smoothed by convolution using a Gaussian filter with a $16 \mathrm{~mm}$ full width at half maximum. The pixel count of PET images was normalized to the mean pixel count of grey matter in each PET image (rest and activation), which was measured using a gray matter probability map (provided by SPM), according to the following equation:

$$
\sum_{i, j, k} I_{i, j, k} \times G_{i, j, k} / \sum_{i, j, k} G_{i, j, k}
$$

Where $I_{i, j, k}$ and $G_{i, j, k}$ are the pixel counts of the PET images and the probability map of gray matter at the $\left(I_{i, j, k}\right)$ th pixel, respectively. The Digital Imaging and Communications in Medicine (.dcm) format was used for all of the data files in the present study, and was converted to the Analyze format using MRIcro software (www.mricro.com; Chris Rorden, Columbia, SC, USA).

Image analysis. The subtraction method was used to analyze the images $(11,14)$. Subtraction of the rest period signal from the activation PET images was performed using the Subtraction ictal single-photon emission computed tomography co-registered to MRI method (14). A metabolic change map was calculated using the following equation:

$$
\left(I_{i c}-I_{i n}\right) / I_{i n} \times 100(\%)
$$

Where $I_{i c}$ and $I_{\text {in }}$ are the normalized rest and activated PET images, respectively. Metabolic changes $>20 \%$ were considered significant. Changes in the metabolic map containing significant pixels were superimposed onto the T1 MRI template $(15,16)$.

\section{Results and Discussion}

A significantly increased uptake of F-18 FDG was observed in the contralateral (left) primary motor cortex (M1), supplementary motor area (SMA), and ipsilateral (right) cerebellum (Figs. 2-4). Increased glucose uptake (metabolic connectivity) indicates increased neuronal activity (17). Therefore, the results shown in Figs. 2-4 indicated increased neuronal activity in the contralateral (left) primary motor cortex (M1), supplementary motor area (SMA) and ipsilateral (right) cerebellum. An increased number of regional blood flow connections between the M1, SMA and cerebellum during motor tasks has previously been demonstrated using MRI $(18,19)$.

The results of the present study align with results from previous MRI studies $(18,19)$, and demonstrated increased glucose metabolism in the contralateral M1, SMA, and ipsilateral cerebellum, using FDG-PET. In the process of neuronal activation, regional metabolic connections precede regional blood flow connections, which are typically measured by fMRI (20). The present results show that it is possible to detect metabolic connectivity using FDG-PET. Furthermore, the present study established a simple imaging procedure for
FDG-PET, thus suggesting that FDG-PET may be considered a reliable neuronal imaging method for analyzing glucose metabolism in the brain.

In summary, the present study used PET to demonstrate increased human motor-associated activation of the brain following administration of FDG, in a subject performing a motor task. In addition, a two-day protocol was established for acquiring detailed images of neuronal activation. FDG-PET-based metabolic connectivity studies may be useful for investigating brain neuronal activation, particularly in motor neuronal activation.

\section{Acknowledgements}

This study was supported by a grant from Korea University (no. K1507831).

\section{References}

1. Lotze M, Montoya P, Erb M, Hülsmann E, Flor H, Klose U, Birbaumer N and Grodd W: Activation of cortical and cerebellar motor areas during executed and imagined hand movements: An fMRI study. J Cogn Neurosci 11: 491-501, 1999.

2. Schreckenberger M, Spetzger U, Sabri O, Meyer PT, Zeggel T, Zimny M, Gilsbach J and Buell U: Localisation of motor areas in brain tumour patients: A comparison of preoperative $[18 \mathrm{~F}]$ FDG-PET and intraoperative cortical electrostimulation. Eur J Nucl Med 28: 1394-1403, 2001.

3. Rorden $\mathrm{C}$ and Karnath HO: Using human brain lesions to infer function: A relic from a past era in the fMRI age? Nat Rev Neurosci 5: 813-819, 2004.

4. Lee DS, Kang H, Kim H, Park H, Oh JS, Lee JS and Lee MC: Metabolic connectivity by interregional correlation analysis using statistical parametric mapping (SPM) and FDG brain PET; methodological development and patterns of metabolic connectivity in adults. Eur J Nucl Med Mol Imaging 35: 1681-1691, 2008.

5. Kelloff GJ, Hoffman JM, Johnson B, Scher HI, Siegel BA Cheng EY, Cheson BD, O'Shaughnessy J, Guyton KZ and Mankoff DA: Progress and promise of FDG-PET imaging for cancer patient management and oncologic drug development. Clin Cancer Res 11: 2785-2808, 2005.

6. Hoh CK: Clinical use of FDF PET. Nucl Med Biol 34: 737-742, 2007.

7. Videbech P: PET measurements of brain glucose metabolism and blood flow in major depressive disorder: A critical review. Acta Psychiatr Scand 101: 11-20, 2000.

8. Varrone A, Asenbaum S, Vander Borght T, Booij J, Nobili F, Nagren K, Darcourt J, Kapucu OL, Tatsch K, Bartenstein P and Van Laere K; European Association of Nuclear Medicine Neuroimaging Committee: EANM procedure guidelines for PET brain imaging using [18F]FDG, version 2. Eur J Nucl Med Mol Imaging 36: 2103-2110, 2009.

9. Daube-Witherspoon ME, Matej S, Karp JS and Lewitt RM: Application of the row action maximum likelihood algorithm with spherical basis functions to clinical PET imaging. IEEE Trans Nucl Sci 48: 24-30, 2001.

10. Lee DS, Lee JS, Kang KW, Jang MJ, Lee SK, Chung JK and Lee MC: Disparity of perfusion and glucose metabolism of epileptogenic zones in temporal lobe epilepsy demonstrated by SPM/SPAM analysis on 150 water PET, [18F]FDG-PET, and [99mTc]-HMPAO SPECT. Epilepsia 42: 1515-1522, 2001.

11. Lee JJ, Kang WJ, Lee DS, Lee JS, Hwang H, Kim KJ, Hwang YS, Chung JK and Lee MC: Diagnostic performance of 18F-FDG PET and ictal 99mTc-HMPAO SPET in pediatric temporal lobe epilepsy: Quantitative analysis by statistical parametric mapping, statistical probabilistic anatomical map, and subtraction ictal SPET. Seizure 14: 213-220, 2005.

12. Choi JY, Lee KH, Na DL, Byun HS, Lee SJ, Kim H, Kwon M, Lee KH and Kim BT: Subcortical aphasia after striatocapsular infarction: Quantitative analysis of brain perfusion SPECT using statistical parametric mapping and a statistical probabilistic anatomic map. J Nucl Med 48: 194-200, 2007. 
13. Lee JS and Lee DS: Analysis of functional brain images using population-based probabilistic atlas. Curr Med Imag Rev 1: 81-87, 2005.

14. Lee SK, Lee SY, Yun CH, Lee HY, Lee JS and Lee DS: Ictal SPECT in neocortical epilepsies: Clinical usefulness and factors affecting the pattern of hyperperfusion. Neuroradiology 48 678-684, 2006

15. Spanaki MV, Spencer SS, Corsi M, MacMullan J, Seibyl J and Zubal IG: Sensitivity and specificity of quantitative difference SPECT analysis in seizure localization. J Nucl Med 40: 730-736, 1999.

16. Avery RA, Spencer SS, Studholme C, Stokking R, Morano G, Corsi M, Seibyl JP, Spencer DD and Zubal IG: Reproducibility of serial peri-ictal single-photon emission tomography difference images in epilepsy patients undergoing surgical resection. Eur J Nucl Med 27: 50-55, 2000.
17. Magistretti PJ and Pellerin L: Cellular mechanisms of brain energy metabolism and their relevance to functional brain imaging. Philos Trans R Soc Lond B Biol Sci 354: 1155-1163, 1999.

18. Arai N, Lu MK, Ugawa Y and Ziemann U: Effective connectivity between human supplementary motor area and primary motor cortex: A paired-coil TMS study. Exp Brain Res 220: 79-87, 2012.

19. Stoodley CJ, Valera EM and Schmahmann JD: Functional topography of the cerebellum for motor and cognitive tasks: An fMRI study. Neuroimage 59: 1560-1570, 2012.

20. Lee DS, Kang H, Kim H, Park H, Oh JS, Lee JS and Lee MC: Metabolic connectivity by interregional correlation analysis using statistical parametric mapping (SPM) and FDG brain PET; methodological development and patterns of metabolic connectivity in adults. Eur J Nucl Med Mol Imaging 35: 1681-1691, 2008. 